\title{
Translational Study of Hydrogen Gas Inhalation as Adjuncts to Reperfusion Therapy for Acute Myocardial Infarction
}

\author{
Hiroshi Asanuma, MD, PhD; Masafumi Kitakaze, MD, PhD
}

$A$ lthough current medical therapies have dramatically improved, heart failure (HF) remains a major cause of morbidity and mortality worldwide, ${ }^{1}$ and importantly, ischemic heart disease such as acute myocardial infarction (AMI) is a leading cause of HF. Therefore, significant clinical effort has been directed towards preventing AMI and reducing the size of the infarction in individuals with AMI. Early reperfusion of the ischemic myocardium by percutaneous coronary intervention (PCI) is the most effective way to reduce final infarct size and improve clinical outcome in patients with AMI. However, in some patients who receive reperfusion therapy, reperfusion per se paradoxically induces myocardial injury known as "reperfusion injury", which blunts the benefits of reperfusion. There has been continued research for better cardioprotective modalities that would reduce myocardial ischemia-reperfusion (IR) injury. However, the strategies to limit reperfusion injury and thus infarct size have not been well implemented in clinical settings. We previously reported that carperitide limited infarct size in a large-scale clinical trial; ${ }^{2}$ however, infarct size was reduced by only $14.7 \%$, and the discovery of other therapeutics to limit infarct size may be clinically useful. However, this mild infarct size-limitation may not contribute largely in the clinical setting, which forces us to further develop new methods to limit infarct size.

Interestingly, hydrogen $\left(\mathrm{H}_{2}\right)$ gas has been reported to provide beneficial therapeutic effects through its reducing capacity, including effects on oxidative stress, inflammation, cell death and metabolism. ${ }^{3}$ It has been reported that $\mathrm{H}_{2}$ gas inhalation (HI) during reperfusion reduced infarct size in a rat model of cardiac IR injury. ${ }^{4}$ Furthermore, it has also been reported that $\mathrm{HI}$ during reperfusion reduced infarct size in a canine model of cardiac IR injury via opening of mitochondrial ATP-sensitive $\mathrm{K}^{+}$channels followed by inhibition of mitochondrial permeability transition pores. ${ }^{5}$ Because the canine experimental model can evaluate the precise systemic hemodynamics and the contraction/ relaxation kinetics resemble those of humans, it can be assumed that HI would limit infarct size in humans (Figure).

In this issue of the Journal, Katsumata et $\mathrm{al}^{\mathbf{6}}$ report on their first clinical study showing that HI during PCI is feasible and safe and may also promote left ventricular (LV) reverse remodeling at 6 months after STEMI in a

\section{Article p 940}

single-center, prospective, open-label, rater-blinded study. In this study, 20 patients with an initial diagnosis of STEMI were assigned to either an $\mathrm{HI}$ group $\left(1.3 \% \mathrm{H}_{2}\right.$ with $26 \%$ oxygen) or a control group ( $26 \%$ oxygen). First of all, they demonstrate there were no HI-related severe adverse events. With regard to the efficacy of the primary outcome,

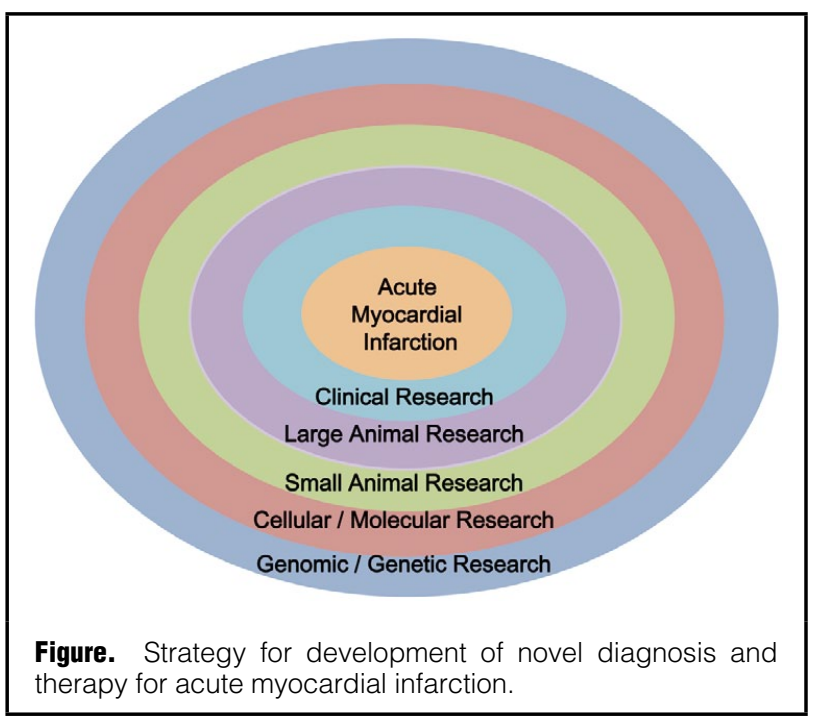

Table. Factors That Prescribes Myocardial Infarct Size
- Reperfusion therapy
- Ischemic preconditioning (prodromal angina)
- Risk area size
- Duration of ischemia (time to treatment)
- Collateral flow
- Age
- Comorbidities
- Effects of medications for comorbidities

The opinions expressed in this article are not necessarily those of the editors or of the Japanese Circulation Society.

Received May 15, 2017; accepted May 15, 2017; released online June 1, 2017

Department of Internal Medicine, Meiji University of Integrative Medicine, Nantan (H.A.); Department of Clinical Research and Development, National Cerebral and Cardiovascular Center, Suita (M.K.), Japan

Mailing address: Masafumi Kitakaze, MD, PhD, Department of Clinical Research and Development, National Cerebral and Cardiovascular Center, 5-7-1 Fujishirodai, Suita 565-8565, Japan. E-mail: kitakaze@zf6.so-net.ne.jp

ISSN-1346-9843 All rights are reserved to the Japanese Circulation Society. For permissions, please e-mail: cj@j-circ.or.jp 
the cardiac salvage index, measured by cardiac magnetic resonance imaging $(\mathrm{CMR})$, there was no statistically significant difference in eligible patients between the HI group and the control group. Secondary outcomes included (1) changes in LV end-systolic volume index (LVESVI), LV end-diastolic volume index (LVEDVI), LV stroke volume index (LVSVI), and LV ejection fraction (LVEF) as assessed by CMR at 7 days and 6 months after primary PCI; (2) angiographic myocardial blush scores; (3) resolution of ST-segment elevation on 12-lead ECG; and (4) the peak plasma level of creatine kinase (CK). As for the efficacy of the secondary outcomes, although there were no significant between-group differences in angiographic myocardial blush scores, resolution of ST-segment elevation on the 12-lead ECG or peak plasma level of CK, both LVSVI and LVEF significantly increased in the HI group, but there was no change in the control group.

There are thousands of articles describing agents that limit infarct size in animal studies, and several agents have been trialed for the prevention or amelioration of reperfusion injuries. However, results have not been consistently satisfactory. Why has such a discrepancy between the animal studies and the clinical studies occurred (Table)? In 1979, Reimer and Jennings reported that the major determinants of infarct size are (1) risk area size, (2) collateral blood flow and (3) duration of ischemia. ${ }^{7}$ In fact, it has been reported that there is a linear relationship between the area at risk and infarct size in patients with AMI following reperfusion with coronary angioplasty, and for patients with a large risk area the benefit of treatment is clearly seen in the clinical setting. ${ }^{8}$ Furthermore, it is reported that the effect of treatment with adenosine is recognized only for patients with anterior MI. ${ }^{9}$ Therefore anterior MI is suitable for evaluation of drug potency, because patients with a large risk area are more likely to obtain the beneficial effects. The coronary collateral circulation has shown benefits regarding not only infarct size but also mortality in patients with AMI by a pooled analysis. ${ }^{10}$ Humans and dogs have much more collateral blood flow than rabbits, rat, pig, sheep and monkeys. On the other hand, brief periods of ischemia that precede sustained ischemia can markedly decrease infarct size, which Murry et al first described as ischemic preconditioning (IP) in a canine experimental model $^{11}$ and has been consistently confirmed in many species and different models of reperfusion injury. However, numerous experimental studies have revealed that IPmediated cardioprotection is markedly suppressed in some pathological conditions such as hypertension, cardiac hypertrophy, hypercholesterolemia, hyperglycemia, and aging. Therefore, one or the combination of these conditions may blunt the actions of IP in the clinical setting. ${ }^{12}$ Recently, Tamura et al demonstrated that $\mathrm{HI}$ in combination with target temperature management is a safe and feasible procedure for patients with post-cardiac arrest syndrome (PCAS) and thus can be considered as a novel therapeutic approach for PCAS patients. ${ }^{13}$ Unfortunately, this study was not powered to test efficacy, because of it was exploratory, aimed at assessing the probability of positive results of large-scale clinical trials. In this sense, the present study is meaningful, and future randomized studies with large samples of patients with first onset of AMI with a proximal left anterior descending occlusion are largely warranted.

\section{Conflict of Interest}

M.K. reports grants from the Japanese Government, grants from Japan Heart Foundation, grants from Japan Cardiovascular Research Foundation, grants and personal fees from Takeda, Astellas, Sanofi, Pfizer, Novartis, Bheringer-Ingerheim, Tanabe-mitubishi, Kyowahakko-kirin, Abott, and Otsuka outside the submitted work; personal fees from Daiichi-sankyo, Ono, Bayer, from Kowa, Dainihonsumitomo, Sawai, MSD, Calpis, Shionogi, Astrazeneca, Asahikasei Med., Novo Nordisk, Fuji-film RI, and Japan Medical Data, outside the submitted work; grants from Nihon Kohden.

\section{References}

1. Greenberg B. Novel therapies for heart failure: Where do they stand? Circ J 2016; 80: 1882-1891.

2. Kitakaze M, Asakura M, Kim J, Shintani Y, Asanuma H, Hamasaki T, et al. Human atrial natriuretic peptide and nicorandil as adjuncts to reperfusion treatment for acute myocardial infarction (J-WIND): Two randomised trials. Lancet 2007; 370: $1483-1493$

3. Ohta S. Molecular hydrogen as a preventive and therapeutic medical gas: Initiation, development and potential of hydrogen medicine. Pharmacol Ther 2014; 144: 1-11.

4. Hayashida K, Sano M, Ohsawa I, Shinmura K, Tamaki K, Kimura K, et al. Inhalation of hydrogen gas reduces infarct size in the rat model of myocardial ischemia-reperfusion injury. Biochem Biophys Res Commun 2008; 373: 30-35.

5. Yoshida A, Asanuma H, Sasaki H, Sanada S, Yamazaki S, Asano Y, et al. H(2) mediates cardioprotection via involvements of K(ATP) channels and permeability transition pores of mitochondria in dogs. Cardiovasc Drugs Ther 2012; 26: 217-226.

6. Katsumata Y, Sano F, Abe T, Tamura T, Fujisawa T, Shiraishi $\mathrm{Y}$, et al. The effects of hydrogen gas inhalation on adverse left ventricular remodeling after percutaneous coronary intervention for ST-Elevated myocardial infarction: First pilot study in humans. Circ J 2017; 81: 940-947.

7. Reimer KA, Jennings RB. The "wavefront phenomenon" of myocardial ischemic cell death. II: Transmural progression of necrosis within the framework of ischemic bed size (myocardium at risk) and collateral flow. Lab Invest 1979; 40: 633-644.

8. Piot C, Croisille P, Staat P, Thibault H, Rioufol G, Mewton N, et al. Effect of cyclosporine on reperfusion injury in acute myocardial infarction. N Engl J Med 2008; 359: 473-481.

9. Mahaffey KW, Puma JA, Barbagelata NA, DiCarli MF, Leesar MA, Browne KF, et al. Adenosine as an adjunct to thrombolytic therapy for acute myocardial infarction: Results of a multicenter, randomized, placebo-controlled trial: The Acute Myocardial Infarction STudy of ADenosine (AMISTAD) trial. $J$ Am Coll Cardiol 1999; 34: 1711-1720.

10. Meier P, Hemingway H, Lansky AJ, Knapp G, Pitt B, Seiler C. The impact of the coronary collateral circulation on mortality: A meta-analysis. Eur Heart J 2012; 33: 614-621.

11. Murry CE, Jennings RB, Reimer KA. Preconditioning with ischemia: A delay of lethal cell injury in ischemic myocardium. Circulation 1986; 74: 1124-1136.

12. Asanuma H, Kitakaze M. Does the treatment of both hypertension and cardiac hypertrophy not only prevent but also treat acute myocardial infarction? Circ J 2011; 75: 1061-1062.

13. Tamura T, Hayashida K, Sano M, Suzuki M, Shibusawa T, Yoshizawa J, et al. Feasibility and safety of hydrogen gas inhalation for post-cardiac arrest syndrome: First-in-human pilot study. Circ J 2016; 80: 1870-1873. 